\title{
Gas sensing properties of Porphyrins-Graphene composite electrospun fibers
}

\author{
J. Avossa ${ }^{1}$, E. Zampetti ${ }^{1}$, G. Scarascia Mugnozza ${ }^{2}$, F. De Cesare ${ }^{2,1}$ \\ R. Paolesse ${ }^{4,1}$, C. Di Natale N $^{3,1}$, A. Macagnano ${ }^{1}$ \\ 1. Institute for Atmospheric Pollution Research-National Research Council, Roma; Italy. \\ 2. DIBAF-University of Tuscia, Viterbo; Italy; \\ 3. Dep. of Electronic Engineering-University of Tor Vergata, Roma; Italy \\ 4. Dep. Of Chemical Science and Technology-University of Tor Vergata, Roma; Italy
}

\begin{abstract}
In this paper, electrospinning technique is used to prepare large surface composite materials where the sensing properties of porphyrins are combined with the conductivity and high reactivity of graphene. Results shows a peculiar sensitivity to small electron donor molecules with an enhanced selectivity towards ammonia and $\mathrm{NO}_{2}$.
\end{abstract}

Key words: gas sensors, electrospinning, porphyrins, graphene, ammonia

\section{Introduction}

In this paper we introduce a novel approach to prepare conductive gas sensors based on the chemical sensitivity of porphyrins. It consists in an electrospun polymeric scaffold enriched with porphyrins, for the chemical selectivity, and graphene flakes, for the electric conductivity. Electrospinning is one of the most versatile and inexpensive manufacturing technologies to design and develop nanostructured sensors to detect gases and volatile organic compounds (VOCs) [1]. Porphyrins are among the most versatile ligand platforms. They can interact with airborne molecules making use of a large spectrum of different mechanisms. This capability has been exploited to develop chemical sensors. Since the scarce conductivity of porphyrins films these sensors are mostly based on mass and optical transducers [2].

The gas sensing properties of the prepared materials depend on the porphyrin to graphene ratio in the composite. This feature enables a fine tuning of the sensor properties that can be used to enhance the performance towards selected targets.

\section{Experimental}

The main components of the composite material were Poly[(R)-3-hydroxybutyric acid, (PHB), polystyrene (192,000 MW, PS), graphene nanopowder $(\leq 5 \mu \mathrm{m}$ size, $\mathrm{G})$, and free-base tetraphenylporphyrins (H2TPP). Suspensions of H2TPP with PHB-PS and increasing concentrations of graphene (in $\mathrm{CHCl} 3)$ were used for Electrospinningdeposition after sonication and stirring. The materials were deposited onto a interdigitated platinum electrodes onto glass substrate. The depositions were carried out in a home-made electrospinning apparatus. The fibrous layers were obtained by applying 3-6 kV of electrostatic DC voltage between the tip of the stainless steel in the syringe pump and the substrate housed on the grounded collector placed about $5 \mathrm{~cm}$ away.

Sensors $(7 \times 11 \mathrm{~mm})$ were placed in a measurement cell $(100 \mathrm{ml})$ and the conductivity was measured during the exposure to known volumes of vapors and gases.

\section{Results and Discussions}

The different fibrous coatings of the substrates were highly porous, showing interconnected void volumes and high surface-to-volume ratios (Fig. 1 and 2). The polymer fibres coated the whole electrodes surface ensuring the electric continuity between the electrodes $(10 \mu \mathrm{m}$ gaps). The conductivity and its dependence on temperature and relative humidity strongly depends on the amount of graphene. On the other hand, increase of polymer concentration facilitates the adhesiveness of the fibers onto the the platinum electrodes.

Porphyrins affected the morphology of the fibers conferring a more homogeneous range of 
the diameter, and, as expected, the sensing properties.

The sensors show a large and rapid response to $\mathrm{NH}_{3}$ and $\mathrm{NO}_{2}$.

Graphene concentration controls the sensor dependence on the humidity and temperature. Specifically, at larger graphene concentration the sensor works at room temperature, it is highly selective to $\mathrm{NH} 3$ and $\mathrm{NO} 2$, but the sensitivity is activated in a humid environment and it is independent from the temperature. At smaller graphene concentration the sensitivity is activated by the temperature, the largest response is obtained at $50^{\circ} \mathrm{C}$ (Fig. 4), and it decreases in humid air. Furthermore, in this condition the selectivity to $\mathrm{NO}_{2}$ respect to $\mathrm{NH}_{3}$ increases with a ratio of sensitivities larger than 600 (Fig. 3).

The chemical interactions between porphyrin and the tested gases were confirmed by UV-Vis spectra where bands were shifted and changed the absorbance intensity.

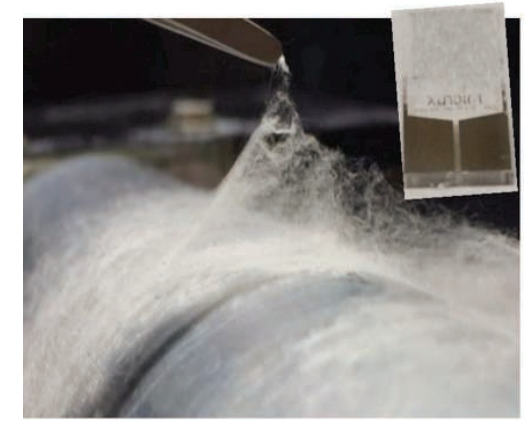

Fig. 1 Picture of the electrospun fibres. In the inset the prepared sensor.

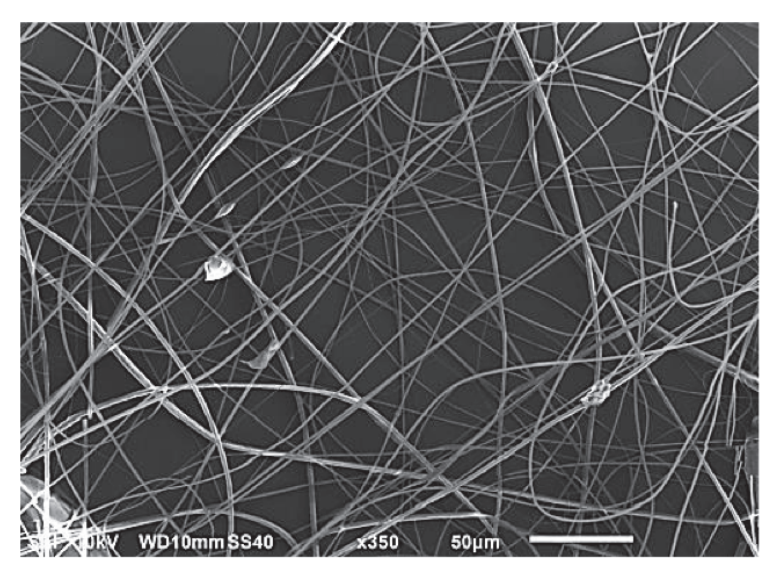

Fig. 2 SEM image of the fibers bundle.

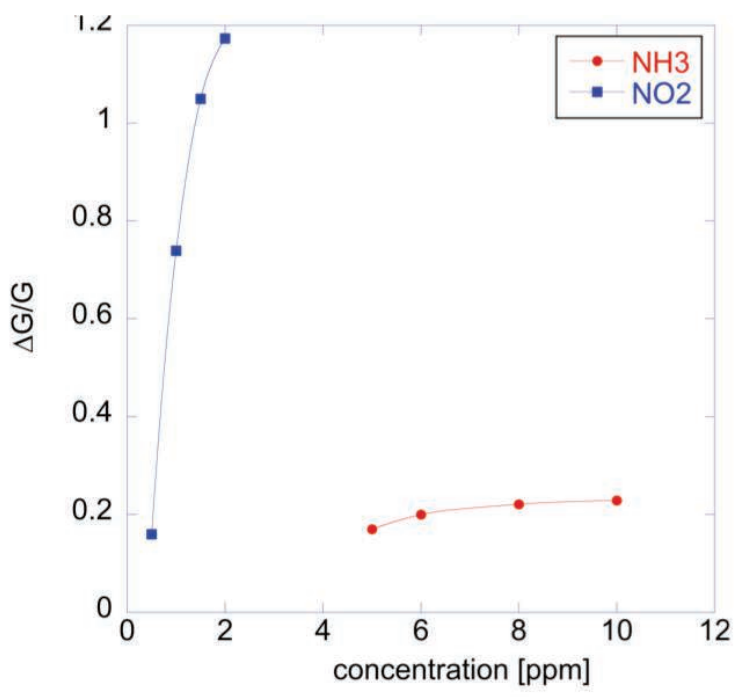

Fig. 3. Relative change of conductivity respect to the concentration of $\mathrm{NO}_{2}$ and $\mathrm{NH}_{3}$. Measurements were taken at $\mathrm{T}=50^{\circ} \mathrm{C}$. The non-linear characteristics suggests a "langmuirian" adsorption process.

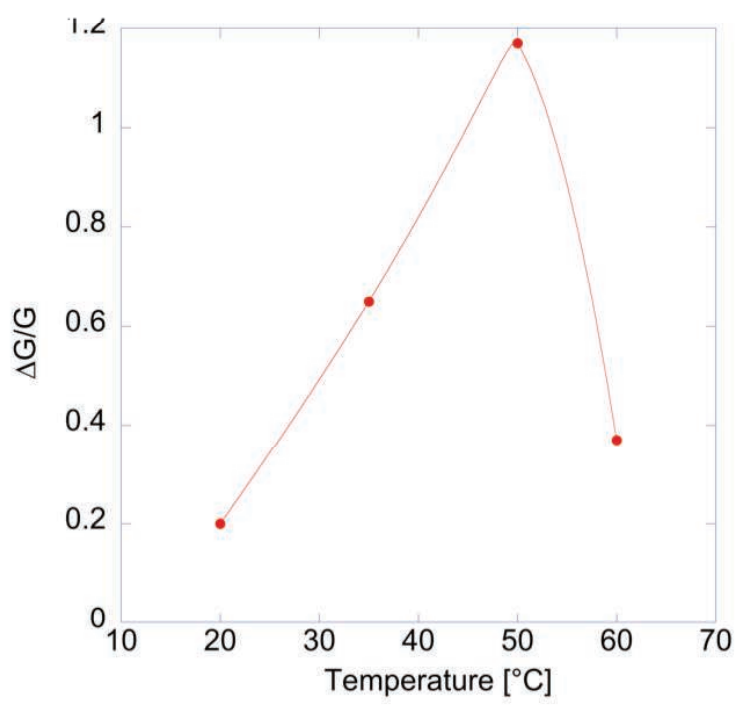

Fig. 4. Influence of sensor temperature to the relative change of conductivity due to the exposure to $2 \mathrm{ppm}$ of $\mathrm{NO}_{2}$.

\section{References}

[1] R. Paolesse, S. Nardis, D. Monti, M. Stefanelli, C. Di Natale, Porphyrinoids for chemical sensors applications. Chem Rev 117, 2517-2583 (2017) doi: 10.1021/acs.chemrev.6b00361

[2] A. Macagnano, E. Zampetti, E. Kny (eds) Electrospinning for High Performance Sensors, Springer International Publishing, 2015 (329 pp), ISBN 978-3-319-14406-1. 